\title{
Fulgides as switches for intramolecular energy transfer
}

\author{
J. Walz, K. Ulrich, H. Port, H.C. Wolf \\ 3. Physikalisches Institut, Universität Stuttgart. D-70550 Stuttgart. Germany
}

\author{
J. Wonner and F. Effenberger \\ Institut für Orgnaische Chemie, Universität Stuttgart, D-70550 Stuttgart, Germany
}

Received 5 August 1993; in final form 11 August 1993

\begin{abstract}
In a molecular unit which is a donor-fulgimide-acceptor triade, the possibility of an intramolecular energy transfer from donor to acceptor depends upon the isomer configuration of the photochromic fulgimide. It is shown that a switching on and off of this intramolecular energy transfer is possible by photoinduced shifting of the fulgimide levels between a trap and an antitrap function.
\end{abstract}

\section{Introduction}

Switching of intramolecular energy transfer is a challenging subject [1-3]. The problem is: is it possible to use a photochromic molecule $\mathrm{F}$ as central part of a supermolecule D-F-A to allow or to hinder energy transfer from $D$ (donor) to A (acceptor) by switching $F$ with light of the appropriate wavelength from one of its photochromic configurations into the other one and back?

Promising candidates for usable photochromic molecules are the fulgides. It is known that molecules of this class can be transformed by light in a reversible way from a configuration $E$ (see fig. 1) into a configuration $C$ [4-6]. Irradiation with UV light causes an intramolecular ring closure reaction (colouring), visible light causes a ring opening reaction (bleaching) [7]. The kinetics of such processes and the stability of educts and products during many cycles have been studied on selected fulgides [8-10]. In order to follow this process step by step, we also investigated the molecules 3 and 4 which are the parts D-F and F-A, respectively, of 5, D-F-A [11-13].

Our first attempts to use the ring opening-ring closure reaction of $F$ ( 1 in scheme 1 ) to interrupt or to close the conjugation in a polyene-like part of molecules D-F or F-A were not successful $[11,12,14]$. The photoreaction during colouring produced sev-

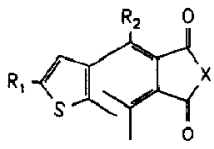

$F_{\mathbf{E}}$
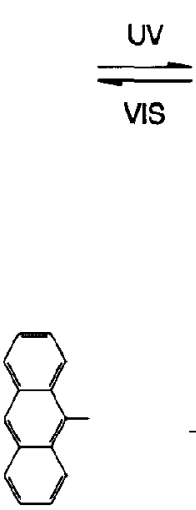

An<smiles>[R]C1CC2C([R])C3C(=O)[X]C(O)C3C(C)(C)C2(C)S1</smiles>

$F_{C}$<smiles>CCCOC(O)[C@@H]1CC2=CC3CCCN4CCCC(C23)[C@@H]1O[C@H]4O</smiles>

ACE

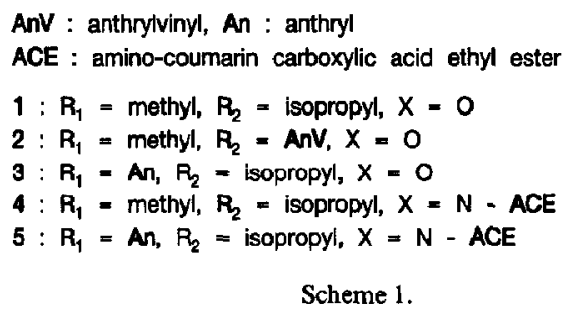

Scheme 1.

eral isomers with overlapping absorption spectra. As a result, selective photobleaching of the desired species was not feasible.

Therefore we tried to realize an alternative model 
for using the photochromism of fulgides for switching on and off an intramolecular transfer of energy from $D$ to $A$. This concept is shown schematically in fig. 1 . In this concept, the molecule $F$ is in its configuration $\mathrm{C}$ a trap, in the configuration $\mathrm{E}$ an antitrap for D-A energy transfer. As a supermolecule for testing this idea we used the molecule 5 . The donor $D$ is the anthryl group which can be excited selectively. As acceptor A we used a coumarin molecule. Due to its high quantum efficiency, fluorescence of $A$ is an indicator for energy transfer from $D$ to $A$ via the fulgimide $\mathrm{F}$ as shown in fig. 1.

\section{Experimental}

Details of the synthesis of the molecules shown in scheme 1 are described elsewhere [12]. For measuring the absorption spectra we used a conventional UV/VIS spectrometer (Pye-Unicam SP8-250). For colouring and bleaching we used a mercury arc $\mathrm{HBO}$ 200 (Osram) and a $0.5 \mathrm{~m}$ monochromator. Emission and excitation spectra were excited by a xenon arc XBO 450 (Osram) in combination with a 0.25 $\mathrm{m}$ double monochromator and detected by a photomultiplier with S 20 cathode in combination with a $1 \mathrm{~m}$ double monochromator. All spectra were cor-
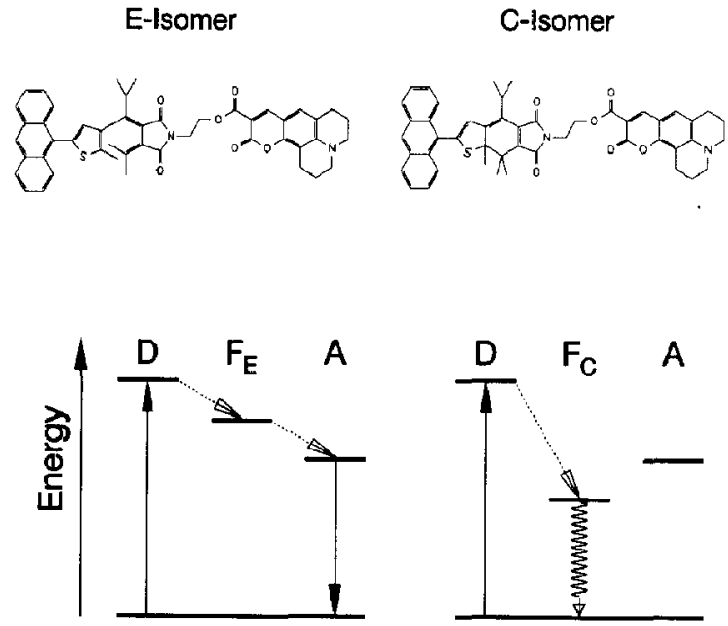

Fig. 1. Concept for switching intramolecular energy transfer in a molecular system D-F-A (molecule 5 as example). In the $\mathrm{E}$ isomer, the acceptor level forms the lowest excited state, but in the $\mathrm{C}$ isomer the fulgimide part is lowest. rected for spectral sensitivity, excitation spectra for spectral distribution of excitation intensity. The spectra were investigated in liquid solution at room temperature. The determination of the reaction quantum yields follows the procedure described in ref. [10].

\section{Results and discussion}

To realize the concept shown in fig. 1 we first investigated the molecules 3 and 4 which are the left part, D-F, and the right part, F-A, respectively, of the final molecule 5. As an example, fig. 2 shows the absorption spectra of 3 during colouring with $\lambda=366$ $\mathrm{nm}$. The photoreaction from the $\mathrm{E}$ to the $\mathrm{C}$ configuration occurs with a quantum yield $\phi_{\mathrm{EC}}=46 \% \pm 7 \%$. $\phi_{\mathrm{EC}}$ does not depend on the excitation wavelength $(\lambda=313,334,366,405 \mathrm{~nm})$. Characteristic for the colouring is the increase of absorption between 450 and $600 \mathrm{~nm}$ which is due to the $C$ configuration. The process is reversible with a quantum yield $\phi_{\mathrm{CE}}=10 \% \pm 3 \%$. Both quantum yiclds are comparable to those of the fulgide without an anthryl group (1).

Fig. 3 shows the absorption spectrum of molecule 5 in its $\mathrm{E}$ configuration. It is a superposition of anthracene absorption (the vibronic structure $S_{0} \rightarrow S_{1}$ between 330 and $400 \mathrm{~nm}$ and the $S_{0} \rightarrow S_{3}$ absorption at $258 \mathrm{~nm}$ ), the fulgimide absorption (below 350 $\mathrm{nm}$ ) and the absorption of coumarin (absorption in the whole range between 230 and $470 \mathrm{~nm}$ with main absorption band at $436 \mathrm{~nm}$ ). The reaction quantum yields for colouring $(\mathrm{E} \rightarrow \mathrm{C})$ and for bleaching $(C \rightarrow E)$ were measured with light of different wavelengths. The quantum yield for colouring $\phi_{\mathrm{EC}}$ depends on the wavelength $\left(\phi_{\mathrm{EC}}=0.18,0.13,0.04,0.01\right.$ for $\lambda=313,334,366,405 \mathrm{~nm}$, respectively). The quantum yield for the process from $\mathrm{C}$ to $\mathrm{E}$ is not easy to determine because the fluorescence light of 5 (see fig. 3 ) is reabsorbed by the $\mathrm{C}$ isomer. By measuring at different concentrations and extrapolation to $c=0$, we obtained $\phi_{C E}=12 \% \pm 4 \%$ independent of the wavelength.

The fluorescence of $\mathbf{5 E}$ is coumarin type and identical with that of $\mathbf{4 E}$. This emission can be used as an indicator for energy transfer from the anthryl group as donor D to the coumarin group as acceptor 


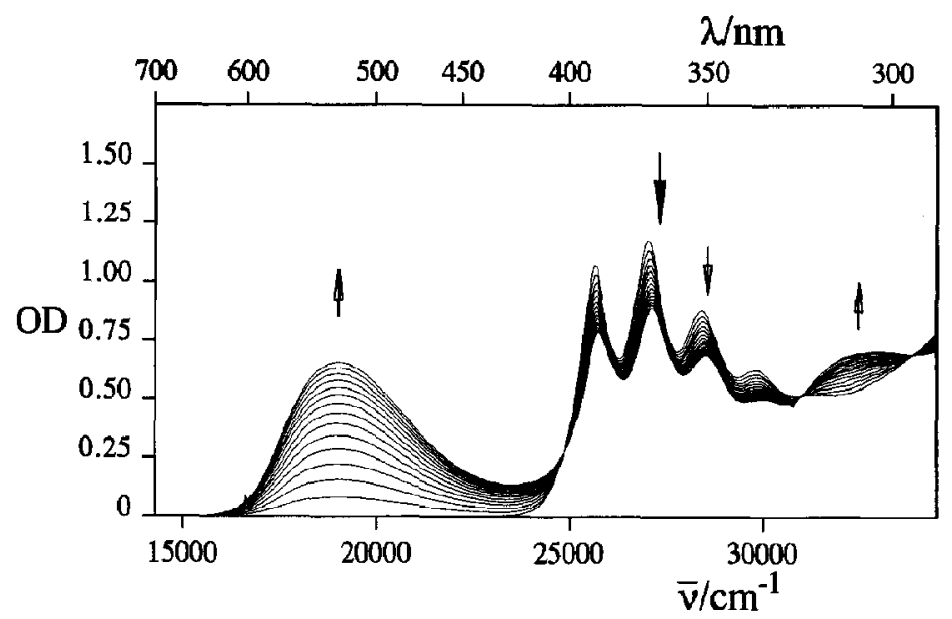

Fig. 2. Colouring of $3 \mathrm{E}$ with $\lambda=366 \mathrm{~nm}$ (bold arrow). The faint arrows indicate decreasing and increasing absorption bands. Measurements in toluene at $T=295 \mathrm{~K}, c=10^{-4} \mathrm{~mol} / \mathrm{e}$.

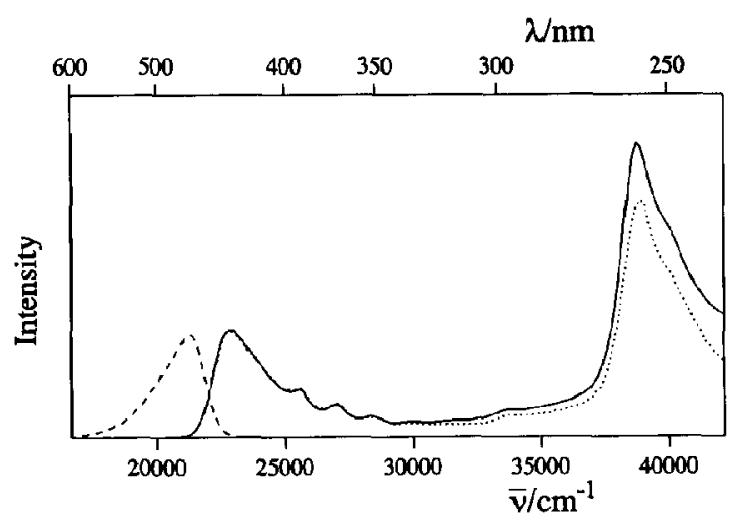

Fig. 3. $5 E$ in methylene chloride at $T=295 \mathrm{~K}$. Absorption (full line) and excitation spectrum detected at $\lambda=500 \mathrm{~nm}$ (dotted line). In addition, the emission (broken line) excited at $\lambda=400$ nm is shown (on the left).

A according to fig. 1. In fig. 3 the fluorescence excitation spectrum of $\mathbf{5 E}$ is shown. It demonstrates that excitation of the anthryl bands, in particular at $\lambda=258 \mathrm{~nm}$, leads to coumarin emission.

Instead, when exciting molecule 5 in its $\mathbf{C}$ configuration with $\lambda=258 \mathrm{~nm}$, the coumarin fluorescence almost disappears (the residual emission is probably due to small amounts of the $E$ isomer). The absence of coumarin emission of the $C$ isomer is interpreted as a result of trapping, i.e. the fulgimide part is a trap for energy absorbed in the donor, and the energy is radiationlessly deactivated by the fulgimide in $\mathbf{5 C}$.

When $\mathbf{5 C}$ is irradiated with light of wavelength $\lambda=520 \mathrm{~nm}$ which is absorbed by the fulgimide part and drives the photoreaction $\mathbf{5 C} \rightarrow \mathbf{5 E}$ (ring opening), the intensity of the fluorescence increases (fig. 4a). Irradiation with light of $\lambda=320 \mathrm{~nm}$ induces ring closure. As a result, the fluorescence intensity decreases. Fig. 4 b shows one switching cycle which can be repeated.

After the first bleaching, the fluorescence is not fully diminished in the consecutive cycles but only to a value which is determined by the achievable photostationary equilibrium for the photoreactions. This means that at all times a mixture of both educt and product molecules exists. A quantitative treatment would have to consider competitive side reactions (to the $Z$ isomer) which are kept small in the isopropyl derivative [10] and could be omitted in the present context.

For the molecular system studied the question about the energy transfer mechanism cannot be answered definitely. Most probably, it can be understood as intramolecular relaxation (internal conversion) to the respective lowest excited state of the supermolecule. For the $C$ isomer this state $\left(F_{C}\right)$ can be populated directly via relaxation within the $D-F$ subunit (see fig. 1) but also - at least in principle indirectly by two-step relaxation via the coumarin. 

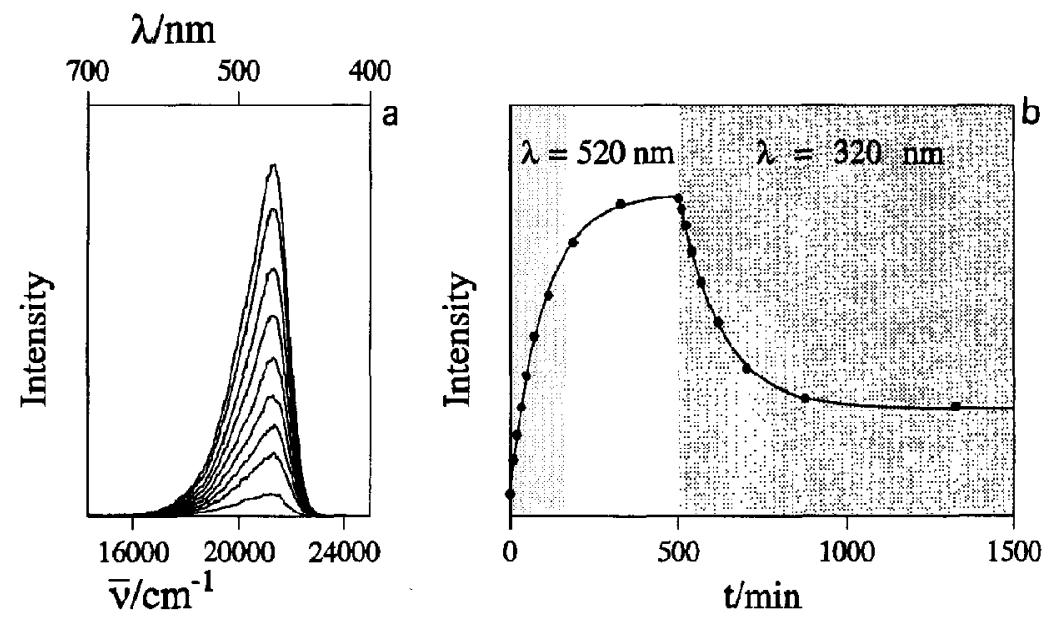

Fig. 4. (a) Coumarin-type fluorescence spectra of $5 C$ observed upon excitation at $400 \mathrm{~nm}$. The stepwise increase of the fluorescence intensity follows consecutive irradiation periods at $\lambda=520 \mathrm{~nm}$ (bleaching).(b) Fluorescence intensity variation during one switching cycle. During the first $500 \mathrm{~nm}, 5 \mathrm{C}$ is bleached $(5 \mathrm{C} \rightarrow 5 \mathrm{E})$ by irradiation at $\lambda=520 \mathrm{~nm}$, after that $5 \mathrm{E}$ is coloured $(5 \mathrm{E} \rightarrow 5 \mathrm{C})$ with $\lambda=320$ nm.

So far, these relaxation pathways cannot be discriminated.

\section{Conclusions}

Apparently, in molecule 5 which is a donor-fulgimide-acceptor triade a switching on and off of an intramolecular energy transfer is possible. The reversible ring opening-ring closure reaction of the fulgimide allows this switching process by shifting the fulgimide levels between trap and antitrap functions.

About the microscopic processes, their efficiencies and dynamics, nothing is known at present. To answer these questions further experiments are under way, including temperature-dependent and ultrafast spectroscopic measurements. The photochromic reaction of the fulgides is known to be in the ps range $[11,15,16]$.

\section{Acknowledgement}

We gratefully acknowledge support from the Deutsche Forschungsgemeinschaft (SFB 329).

\section{References}

[1] F.L. Carter, ed., Molecular electronic devices, Proceedings of the First/Second International Workshop on Molecular Electronic Devices (Marcel Dekker, New York, 1982/ 1984).

[2] J.-M. Lehn, Angew. Chem. 102 (1990) 1347; Angew. Chem. Intern. Ed. Engl. 29 ( 1990$) 1304$.

[3] G. Blessing, N. Holl, H. Port, H.C. Wolf, F. Effenberger and T. Kesmarszky, Mol. Cryst. Liquid Cryst. 183 (1990) 21.

[4] H.G. Heller and S. Oliver, J. Chem. Soc. Perkin Trans. I (1981) 197.

[5] P.J. Darcy, H.G. Heller, P.J. Strydom and J, Whittall, J. Chem. Soc. Perkin Trans. I (1981) 202.

[6] J. Whittall, in: Photochromism - molecules and systems, eds. H. Dürr and H. Bouas-Laurent (Elsevier, Amsterdam, 1990).

[7] H.G. Heller and R.J. Hart, J. Chem. Soc. Perkin Trans. I (1972) 1321.

[8] K. Ulrich, H. Port and P. Bäuerle, Chem. Phys. Letters 155 (1989) 437.

[9] K. Ulrich and H. Por, J. Mol. Struct. 218 (1990) 45.

[10] K. Ulrich, H. Port, H.C. Wolf, J. Wonner, F. Effenberger and H.-D. Ilge, Chem. Phys. 154 (1991) 311.

[11] K. Ulrich, Dissertation, Universität Stuttgart (1991).

[12] J. Wonner, Dissertation, Universität Stuttgart (1992).

[13] J. Walz, Diplomarbeit, Universität Stuttgart (1993).

[14] F. Effenberger and J. Wonner, Chem. Ber. 125 (1992) 2583.

[15] S. Kurita, A. Kashiwagi, Y. Kurita, H. Miyasaka and N. Mataga, Chem. Phys. Letters 171 (1990) 553.

[16] H.-D. Ilge, J. Suehnel, D. Khechinashvili and M. Kaschke, J. Photochem. 38 (1987) 189. 